\title{
A new method to specify the mitigated acoustic test conditions from flight telemetry based on the equivalence of vibro-acoustic response
}

\author{
Shingo Shimazaki ${ }^{1}$ (D) Qinzhong Shi ${ }^{1}$
}

Received: 30 August 2021 / Revised: 3 November 2021 / Accepted: 30 November 2021 / Published online: 17 December 2021

(c) The Author(s) 2021

\begin{abstract}
Ground acoustic tests using stationary sound pressure level spectrum have been conducted to verify the spacecraft survivability against acoustic environment acting on a spacecraft during launch, which is a non-stationary and random dynamic load. In general, a stationary spectrum used in ground acoustic test is traditionally determined by a method called maximax spectrum, which is the enveloped spectrum of time varying non-stationary short-time Fourier transform. However, the maximax spectrum is more or less an excessively conservative test condition because this spectrum focuses on processing of a time-varying acoustic signal itself to extract maximum value, rather than on how the vibro-acoustic response of an excited structure is. In this paper, a new method is proposed to specify a stationary spectrum equivalent to a structural vibro-acoustic response under a non-stationary and random acoustic environment based on extreme response spectrum and fatigue damage spectrum. This proposed method was applied to flight telemetry of both liquid- and solid-propellant launch vehicles developed by JAXA, to show its effect to mitigate the acoustic test conditions compared to the maximax spectrum while maintaining the equivalence of the structural vibro-acoustic response. Furthermore, the maximum predicted environment, which is the statistical upper percentiles of the flight telemetry of eight liquid-propellant launch vehicles, by the proposed method achieved a mitigation of about 2.5 and $6.8 \mathrm{~dB}$ in the extreme stress and cumulative fatigue, respectively, compared to the that which is calculated by the conventional maximax spectrum.
\end{abstract}

Keywords Spacecraft · Acoustic test $\cdot$ Maximax spectrum $\cdot$ Flight telemetry $\cdot$ Extreme response spectrum (ERS) $\cdot$ Fatigue damage spectrum (FDS)

\section{List of symbols}

$A, B, C, D, E, b, c, k, X_{1}, X_{2}$ Coefficients [-]

$C_{\mathrm{np}}$

$D_{\mathrm{f}}$

ERS

FDS

$F_{\mathrm{S}}$

$f_{\mathrm{c}}$

$f_{\text {min }}$

Shingo Shimazaki

shimazaki.shingo@jaxa.jp

1 Japan Aerospace Exploration Agency, Tsukuba Space

Center, Tsukuba-shi, Ibaraki 305-8505, Japan $f_{n}$

$\widehat{H}, \hat{h}$

$m$

$n_{i}$

$N_{i}$

Extreme response spectrum $[\mathrm{m} / \mathrm{s}]$

Fatigue damage spectrum [-]

Sampling rate $[\mathrm{Hz}]$

High-pass cut-off frequency $\quad$ PSD $_{p}$

[Hz]

Lower limit of analysis fre- $\quad p_{\mathrm{f}}$

quency $[\mathrm{Hz}]$

\section{$Q$}

$S, S_{i}$

$T$

$T_{\mathrm{w}}$

$T_{0}$

$v$
Natural frequency of $n$-th mode $[\mathrm{Hz}]$

Transfer function [-]

Mass [kg]

Cycle counts [-]

Number of cycles to failure

[-]

Number of data (sample size)

[-]

Power spectrum density of sound pressure $\left[\mathrm{Pa}^{2} / \mathrm{Hz}\right]$

Sound pressure $[\mathrm{Pa}]$

Quality factor [-]

Surface area $\left[\mathrm{m}^{2}\right]$

Stress [Pa]

Time span [s]

Time window length [s]

Test duration [s]

Mode velocity $[\mathrm{m} / \mathrm{s}]$ 


$\begin{array}{ll}\ddot{x}, \dot{x}, x & \begin{array}{l}\text { Acceleration, velocity, and } \\ \text { displacement }\left[\mathrm{m} / \mathrm{s}^{2}\right],[\mathrm{m} / \mathrm{s}],\end{array} \\ & {[\mathrm{m}]} \\ \mathcal{Z}[] & Z \text {-Transform [-] } \\ \alpha & \text { Probability threshold [-] } \\ \Gamma & \text { Gamma function [-] } \\ \Delta S & \text { Infinitesimal stress [Pa] } \\ \Delta t & \text { Discretized time increment [s] } \\ \zeta & \text { Damping ratio [-] } \\ \sigma & \text { Standard deviation of random } \\ & \text { velocity response [m/s] } \\ \varphi_{n} & \text { Structural mode shape [-] } \\ \phi_{\mathrm{p}} & \text { Acoustic mode shape [-] } \\ \omega_{\mathrm{d}} & \text { Damped natural angular fre- } \\ & \text { quency [rad/s] } \\ \omega_{n} & \text { Natural angular frequency of } \\ & n \text {-th mode [rad/s] }\end{array}$

\section{Introduction}

Ground acoustic tests using stationary sound pressure level (SPL) spectrum have been conducted to verify the spacecraft survivability against acoustic environment during launch as shown in Fig. 1.

In general, a stationary spectrum for ground acoustic test condition is defined based on data processing of the flight telemetry. The acoustic environment impinging on a spacecraft during launch is a dynamic load, and there are many different ways to describe such signals, as shown below [1]:

(a) representative time histories;

(b) average values; (c) line spectrum, auto spectrum, wave-number spectra, and $1 / 3$ octave band spectra for stationary environments;

(d) maximax spectrum for non-stationary environments, and

(e) Fourier spectrum, energy spectrum, and shock response spectrum for transient environments.

Figure 2 shows an example of sound pressure telemetry obtained inside fairing of liquid-propellant launch vehicle during lift-off till solid rocket booster (SRB) burn out. According to this figure, it can be seen that the acoustic environment is a non-stationary and random signal. Therefore, in data processing of flight telemetry for stationary acoustic test condition, among above ways, (d) maximax spectrum have been used conventionally for decades (see e.g. [1-4]).

The maximax spectrum is derived by extracting the maximum values of each frequency bandwidth of a time-varying spectrum based on short-time Fourier transform (STFT), by stationary approximation in time segment divided by a window length, regardless of the time when the maximum value occurs. Therefore, the resulting spectrum depends on processing parameters such as the time window length of the STFT as shown in Fig. 3, calculated from the data in Fig. 2. In this example, the overall (O.A.) difference is about $2.7 \mathrm{~dB}$ between $T_{\mathrm{w}}=0.1 \mathrm{~s}$ and $T_{\mathrm{w}}=1.0 \mathrm{~s}$, and about $5.3 \mathrm{~dB}$ between $T_{\mathrm{w}}=1.0 \mathrm{~s}$ and $T_{\mathrm{w}}=10.0 \mathrm{~s}$. The maximax spectrum is not related to structural vibro-acoustic response of a spacecraft. Consequently, the maximax spectrum has been provided an over-conservative acoustic test condition [5-8]. For example, in reference [7], a comparison of the maximax spectrum and damage-based approach based on flight telemetry of the Titan 4A launch vehicle shows that
Fig. 1 Example of ground acoustic test for spacecraft (in acoustic chamber)

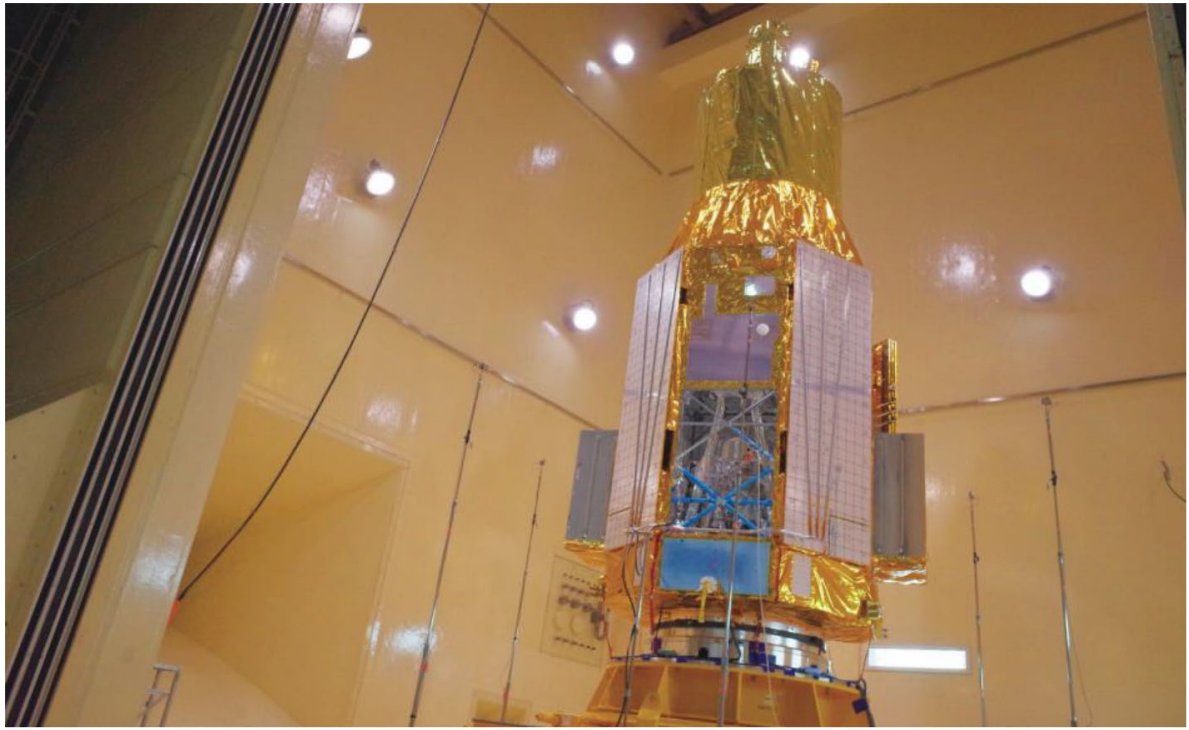


Fig. 2 Example of sound pressure telemetry obtained inside fairing of liquid-propellant launch vehicle

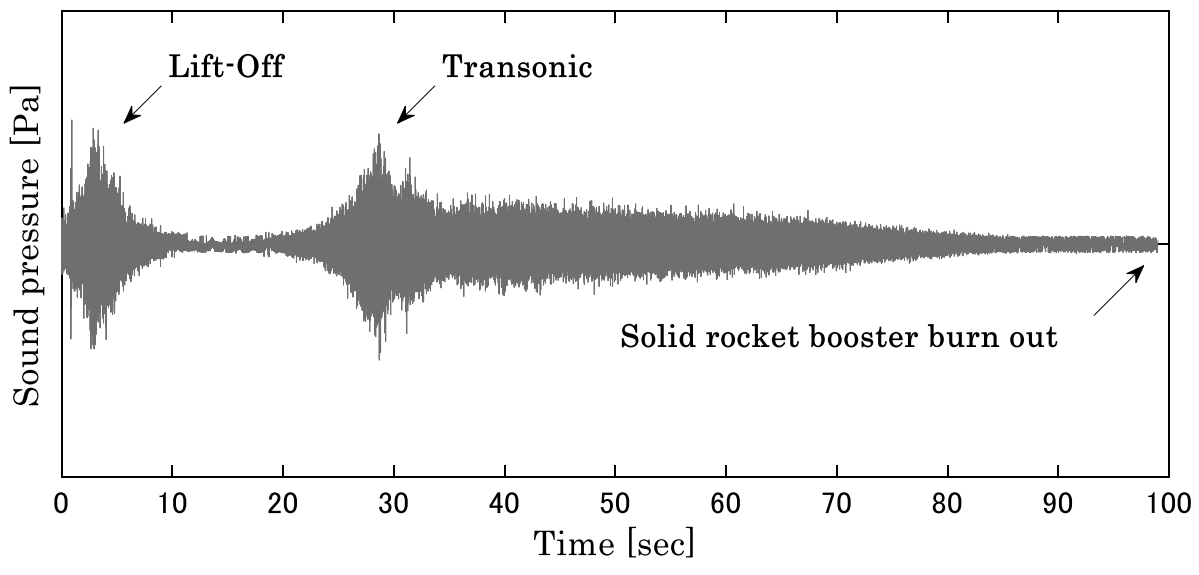

Fig. 3 Analysis examples based on the maximax spectrum with different time window length (overlaps $=50 \%)$

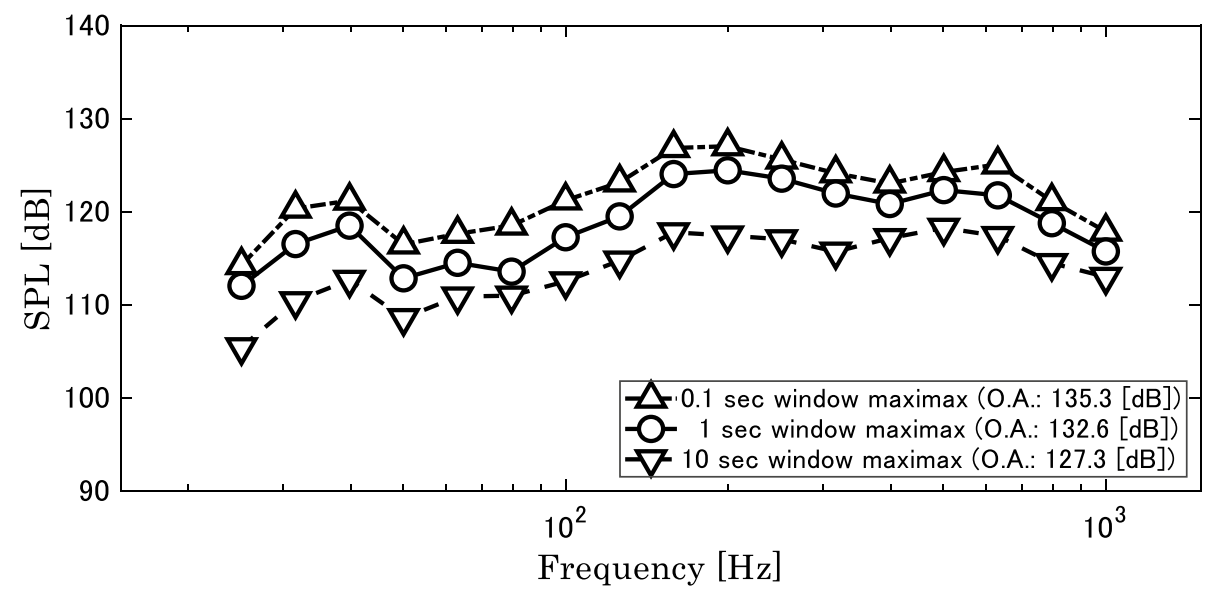

the conventional maximax spectrum may significantly overestimate, especially in the frequency range below $200 \mathrm{~Hz}$.

On the other hand, data processing for transient signals such as pyro-shock calculates the maximum values versus frequency based on shock response spectrum (SRS) by a hypothetical single degree-of-freedom (SDOF) model rather than Fourier transform (see, e.g., [9]). Lalanne applied this model to propose a method to calculate extreme response spectrum (ERS) and fatigue damage spectrum (FDS), for the assessment of base excitation severity from the viewpoint of structural vibration response [10-12]. There were papers based on these spectra, to specify a stationary acceleration spectrum to be equivalent to the extreme stress and cumulative fatigue of SDOF model under a non-stationary base excitation environment (see e.g., [13-15]). However, in order to apply this base excitation concept to the acoustic excitation, a new SDOF model for equivalent ERS and FDS under the acoustic excitation needs to be re-constructed.

Therefore, in this paper, a new method is proposed to specify a stationary spectrum equivalent to a structural vibro-acoustic response under a non-stationary and random acoustic environment based on ERS and FDS, respectively. The novelty of the proposed approach is that it synthesises the damage-based approach to acoustic environments and the derivation of equivalent spectrum for base excitation, both of which have been proposed in previous studies, and applies them to the derivation of equivalent spectrum for acoustic environments. The proposed method first calculates time domain stress response of SDOF model under a non-stationary and random acoustic environment by assuming that structural stress is proportional to mode velocity response [16], where extreme stress and cumulative fatigue are calculated based on ERS and FDS. Then, based on the calculated ERS and FDS, the equivalent stationary spectrum to the extreme stress and cumulative fatigue under a nonstationary acoustic excitation is calculated. Furthermore, the effectiveness of the proposed method is shown by the examples applied to the flight telemetry of both liquid- and solid-propellant launch vehicles developed by JAXA.

\section{Methodology}

Conceptual figure of new SDOF systems composed by multiple parallel SDOF models to specify a stationary acoustic test condition equivalent to a non-stationary and 
Acoustic excitation

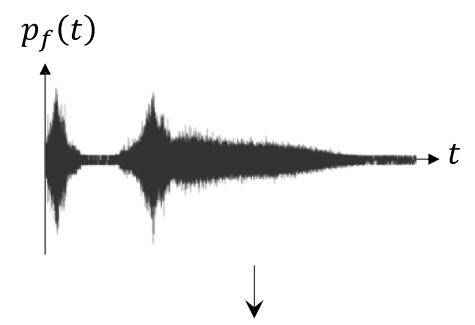

SDOF systems
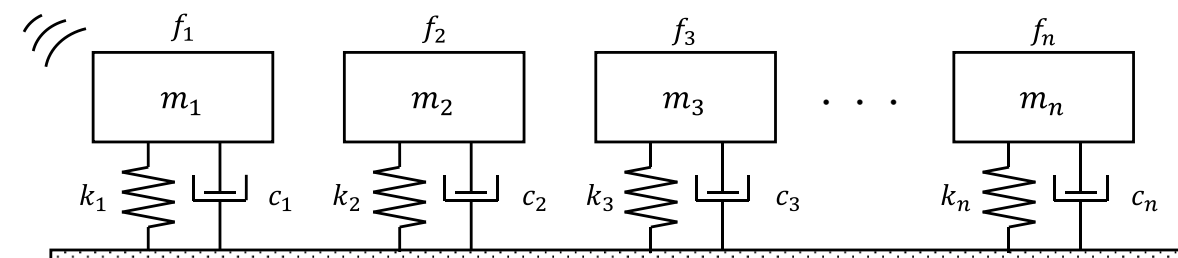

Vibro-acoustic response of each system
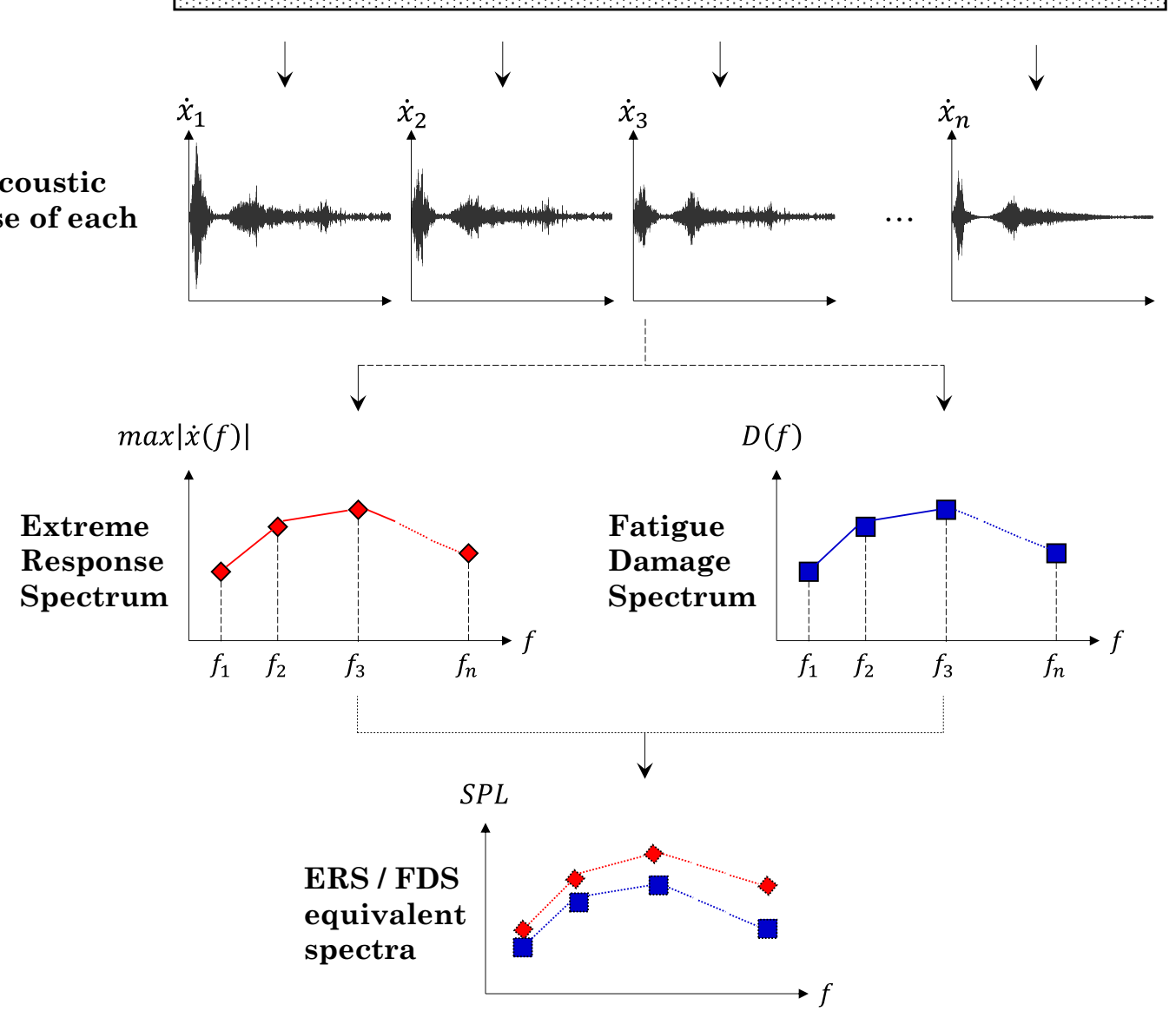

Fig. 4 Conceptual figure of new SDOF systems to specify the stationary acoustic test condition based on ERS and FDS

random acoustic excitation $p_{\mathrm{f}}(t)$ is illustrated by Fig. 4 . Section 2.1 shows the method to calculate vibro-acoustic response of each SDOF model and extraction of ERS and FDS at each model frequencies, from non-stationary and random acoustic signals. Section 2.2 shows the method to specify stationary acoustic test conditions from calculated ERS and FDS.
2.1 Computation of ERS and FDS based on time domain response of SDOF systems under a non-stationary and random acoustic excitation

Equation of motion in time domain of each SDOF system under the sound pressure $p_{f}(t)$ is expressed by Eq. (1) (see 
e.g., [17]) as follows:

$\ddot{x}(t)+2 \zeta \omega_{n} \dot{x}(t)+\omega_{n}^{2} x(t)=C_{\mathrm{np}} \frac{S_{\mathrm{a}}}{m} \cdot p_{\mathrm{f}}(t)$,

where $m, \zeta(=1 /(2 Q)), \omega_{n}\left(=2 \pi f_{n}\right), S_{\mathrm{a}}, \dot{x}(t)$, and $\ddot{x}(t)$ are mass, damping ratio, natural angular frequency of $n$-th mode of multiple SDOF model, surface area, first derivative of displacement and second derivative of displacement, respectively. Besides, $C_{\mathrm{np}}$ is a parameter called joint acceptance, which expresses the structure mode participation under an acoustic excitation, an integral of acoustic mode shape $\phi_{\mathrm{p}}$ and structural vibration mode shape $\varphi_{n}$ calculated by Eq. (2) as follows:

$C_{\text {np }} \equiv \frac{1}{S_{\mathrm{a}}} \int_{S_{\mathrm{a}}} \phi_{\mathrm{p}} \varphi_{n} \mathrm{~d} S_{\mathrm{a}}$

In this paper, to simplify the computation without loss of generality, $C_{\mathrm{np}}=1$ and $\left(S_{\mathrm{a}} / \mathrm{m}\right)=1$. Namely, the acoustic mode shapes (spatial distribution) and structural mode shapes may be arbitrary because these parameters are used back again when calculating the equivalent acoustic spectrum.

According to the Hunt theorem, structural maximum stress $S_{i}$ is proportional to mode velocity $\dot{x}_{i}$, with constant $k$ depending on structural geometric shape and stress concentration factor [16] shown as follows:

$S_{i}=k \dot{x}_{i}$

Thus, Eq. (1) is solved for $\dot{x}(t)$ in stead of $S_{i}$ by the following convolution integration as shown in Eq. (4):

$\dot{x}(t)=\int_{0}^{t} p_{\mathrm{f}}(\tau) \mathrm{e}^{-\zeta \omega_{n}(t-\tau)}\left[\frac{\zeta \omega_{n}}{\omega_{\mathrm{d}}} \sin \left\{\omega_{\mathrm{d}}(t-\tau)\right\}-\cos \left\{\omega_{\mathrm{d}}(t-\tau)\right\}\right] \mathrm{d} \tau$,

where

$\omega_{\mathrm{d}}=\sqrt{1-\zeta^{2}} \omega_{n}$

In the part of integrand of Eq. (4), variable is replaced as shown in Eq. (6) as follows:

$\widehat{h}(t)=\mathrm{e}^{-\zeta \omega_{n} t}\left[\frac{\zeta \omega_{n}}{\omega_{\mathrm{d}}} \sin \left(\omega_{\mathrm{d}} t\right)-\cos \left(\omega_{\mathrm{d}} t\right)\right]$,

where $\alpha$ and $\beta$ are defined as the following Eqs. (7-8):

$X_{1}=\frac{\zeta_{n} \omega_{n}}{\omega_{\mathrm{d}}}$

$X_{2}=-1$.
From this, Eq. (6) can be rewritten as Eq. (9) as follows:

$\widehat{h}(t)=\mathrm{e}^{-\zeta_{n} \omega_{n} t}\left[X_{1} \sin \left(\omega_{\mathrm{d}} t\right)+X_{2} \cos \left(\omega_{\mathrm{d}} t\right)\right]$.

Consequently, suppose discretized time increment is $\Delta t$, $z$-transform of $\hat{h}(t)$ is given by the following:

$\mathcal{Z}[\hat{h}(t)]=X_{1} \mathcal{Z}\left[e^{-\zeta_{n} \omega_{n} \Delta t} \sin \left(\omega_{\mathrm{d}} \Delta t\right)\right]+X_{2} \mathcal{Z}\left[\mathrm{e}^{-\zeta_{n} \omega_{n} \Delta t} \cos \left(\omega_{\mathrm{d}} \Delta t\right)\right]$

$=\frac{X_{1} \mathrm{e}^{-\zeta_{n} \omega_{n} \Delta t} \sin \left(\omega_{\mathrm{d}} \Delta t\right) z}{z^{2}-2 \mathrm{e}^{-\zeta_{n} \omega_{n} \Delta t} \cos \left\{\omega_{\mathrm{d}} \Delta t\right\} z+\mathrm{e}^{-2 \zeta_{n} \omega_{n} \Delta t}}$

$+\frac{X_{2} z\left(z-\mathrm{e}^{-\zeta_{n} \omega_{n} \Delta t}\right) \cos \left(\omega_{\mathrm{d}} \Delta t\right)}{z^{2}-2 \mathrm{e}^{-\zeta_{n} \omega_{n} \Delta t} \cos \left\{\omega_{\mathrm{d}} \Delta t\right\} z+\mathrm{e}^{-2 \zeta_{n} \omega_{n} \Delta t}}$.

$\therefore \mathcal{Z}[\hat{h}(t)]=\frac{X_{2} z^{2}+z \mathrm{e}^{-\zeta_{n} \omega_{n} \Delta t}\left\{X_{1} \sin \left(\omega_{\mathrm{d}} \Delta t\right)-X_{2} \cos \left(\omega_{\mathrm{d}} \Delta t\right)\right\}}{z^{2}-2 \mathrm{e}^{-\zeta_{n} \omega_{n} \Delta t} \cos \left\{\omega_{\mathrm{d}} \Delta t\right\} z+\mathrm{e}^{-2 \zeta_{n} \omega_{n} \Delta t}}$.

Using coefficients $A-E$, $z$-transform of $\widehat{h}(t)$ can be described as Eq. (11) as follows:

$\hat{H}(z)=Z[\hat{h}(t)]=\frac{1}{\Delta t} \cdot \frac{C z^{2}+D z+E}{z^{2}-A z-B}$.

Right-side coefficients of Eq. (11) can be expressed as Eq. (12) by the following comparison between Eqs. (10) and (11):

$A=2 \mathrm{e}^{-\zeta \omega_{n} \Delta t} \cos \left(\omega_{\mathrm{d}} \Delta t\right)$,

$B=-\mathrm{e}^{-2 \zeta \omega_{n} \Delta t}$,

$C=-\Delta t$,

$$
D=\Delta t \cdot \mathrm{e}^{-\zeta \omega_{n} \Delta t}\left\{\left(\zeta \omega_{n} / \omega_{\mathrm{d}}\right) \sin \left(\omega_{\mathrm{d}} \Delta t\right)+\cos \left(\omega_{\mathrm{d}} \Delta t\right)\right\},
$$
and

$E=0$.

Using these coefficients, discrete velocity response $\dot{x}(j)$ $\left(j=3,4, \cdots, N_{\mathrm{d}} . N_{\mathrm{d}}\right.$ is the number of data (sample size)) obtained by sequential computation as Eq. (13) as follows:

$\dot{x}(j)=A \dot{x}(j-1)+B \dot{x}(j-2)+C p_{\mathrm{f}}(j)+D p_{\mathrm{f}}(j-1)+E p_{\mathrm{f}}(j-2)$,

where $x(j=1)=0$ and $\dot{x}(j=2)=0$. ERS is the maximum value of mode velocity in time domain of each SDOF velocity response, corresponding to resonant frequency as follows: 
$\operatorname{ERS}\left(f_{n}\right)=\max \left[\left|\dot{x}\left(t, f_{n}\right)\right|\right]$,

where max[] expresses the function to take the maximum value in [ ].

Cycle counts $n_{i}\left(f_{n}\right)$ to calculate cumulative fatigue are obtained using velocity response $\dot{x}(t)$ in each $f_{n}$. Although there are many different ways to calculate cycle counts such as Peak-valley, Level cross, and Range method, in this paper, Rainflow method that is widely used in cumulative damage evaluation is adopted by using "rainflow" function in numerical analysis software MATLAB ${ }^{\circledR}$ [18]. Famous SN curve in material fatigue theory that expresses the relationship of the magnitude of an alternating stress versus the number of cycles to failure is used as follows to calculate cumulative fatigue damage by constants $b$ and $c$, which are material dependent parameters:

$N_{i}=c S_{i}^{-b}$.

Cumulative fatigue damage $D_{\mathrm{f}}$ is calculated by wellknown Miner's rule as Eq. (16) [19] as follows:

$D_{\mathrm{f}}=\sum_{i} \frac{n_{i}}{N_{i}}$

where $n_{i}$ expresses the number of cycle counts. By inserting Eqs. (15) and (3) into (16), Eq. (16) can be rewritten as follows:

$D_{\mathrm{f}}=\sum_{i} \frac{n_{i}}{c\left(k v_{i}\right)^{-b}}=\frac{k^{b}}{c} \sum_{i} n_{i} v_{i}^{b}$.

Note that the mode velocity $\dot{x}$ is replaced by $v_{i}$. Replacing variable $n_{i}$ and $v_{i}$ as a function of $f_{n}$, FDS is given by the following:

$\operatorname{FDS}\left(f_{n}\right)=\frac{k^{b}}{c} \sum_{i} n_{i}\left(f_{n}\right) \cdot\left\{v_{i}\left(f_{n}\right)\right\}^{b}$.

Noting that FDS in Eq. (18) is function of $\mathrm{k}$ and c, we can assume that the $k=1$ and $c=1$ for simplification of computation without loss the generality because the computation result will not change for the purpose of equivalence between non-stationary and stationary acoustic excitation to the same structure.

\subsection{Specification of stationary acoustic test conditions based on ERS and FDS}

Stationary acoustic test conditions equivalent to the extreme stress and cumulative fatigue under a non-stationary and random acoustic excitation are specified based on calculated ERS and FDS.

Test duration $T_{0}$ is made to be satisfied as the following condition because $T_{0}$ must be greater than reciprocal of lower limit of analysis frequency $f_{\min }$ :

$T_{0} \geq \frac{1}{f_{\min }}$.

On the assumption that peak vibration response distribution of narrow band stationary-random process has the Rayleigh distribution [20], peak velocity response exceeding the ERS with probability $\alpha$ can be derived by the following probability density function:

$$
\begin{aligned}
\operatorname{Pr}[v \leq \mathrm{ERS}] & =\int_{-\infty}^{\mathrm{ERS}} \frac{v^{2}}{2 \sigma_{\mathrm{ERS}}{ }^{2}} \exp \left(-\frac{v^{2}}{2 \sigma_{\mathrm{ERS}}{ }^{2}}\right) \mathrm{d} v \\
& =1-\exp \left(-\frac{\mathrm{ERS}^{2}}{2 \sigma_{\mathrm{ERS}}{ }^{2}}\right) .
\end{aligned}
$$

where $\operatorname{Pr}[]$ expresses probability of [ ] event, and $\sigma_{\mathrm{ERS}}$ is standard deviation of velocity response $v$. Probability that velocity response exceeding ERS never happens $(v \leq$ ERS) by $f_{n} \times T_{0}$ times independent observations, is expressed as follows:

$\operatorname{Pr}\left[v \leq \mathrm{ERS}_{f_{n} T_{0}}=\left\{1-\exp \left(-\frac{\mathrm{ERS}^{2}}{2 \sigma_{\mathrm{ERS}}{ }^{2}}\right)\right\}^{f_{n} T_{0}}\right.$.

From the complementary event of Eq. (21), occurrence probability that peak velocity response exceeding ERS at least once $\operatorname{Pr}[v>\mathrm{ERS}]_{f_{n} T_{0}}$ is expressed as follows:

$$
\begin{aligned}
\operatorname{Pr}[v>\mathrm{ERS}]_{f_{n} T_{0}} & =1-\operatorname{Pr}[v \leq \mathrm{ERS}]_{f_{n} T_{0}} \\
& =1-\left\{1-\exp \left(-\frac{\mathrm{ERS}^{2}}{2 \sigma_{\mathrm{ERS}^{2}}}\right)\right\}^{f_{n} T_{0}} .
\end{aligned}
$$

The probability of $v>$ ERS is expressed by Eq. (23) as follows in case a threshold $\alpha$ is assigned:

$\operatorname{Pr}\left[v>\mathrm{ERS}_{f_{n} T_{0}}=1-\left\{1-\exp \left(-\frac{\mathrm{ERS}^{2}}{2 \sigma_{\mathrm{ERS}^{2}}}\right)\right\}^{f_{n} T_{0}} \geq \gamma\right.$

Therefore, $\sigma_{\mathrm{ERS}}$ is given by the following:

$\sigma_{\mathrm{ERS}}\left(f_{n}\right)=\frac{\operatorname{ERS}\left(f_{n}\right)}{\sqrt{2 \ln \frac{1}{1-(1-\alpha)^{\frac{1}{f_{n} T_{0}}}}}}$.

According to proportional relationship between mode velocity to stress expressed by Eq. (3), peak distribution of stress $f(S)$ can be expressed as follows probability density function: 
$f(S)=\frac{S}{\sigma_{S}^{2}} \exp \left(-\frac{S^{2}}{2 \sigma_{S}^{2}}\right)$,

where $\sigma_{S}$ is standard deviation of stress $S$. Expected value of the number of cycle counts $n_{i}$ can be expressed as follows by using number of observations $f_{n} T_{0}$, probability density $f\left(S_{i}\right)$, and its infinitesimal interval $\Delta S$ :

$n_{i}=f_{n} T_{0} \times f\left(S_{i}\right) \times \Delta S$.

Inserting this in Miner's rule, cumulative fatigue damage $D_{\mathrm{f}}$ is expressed as follows:

$$
\begin{aligned}
D_{\mathrm{f}}=\frac{1}{c} \sum_{i} n_{i} S_{i}^{b} & =\frac{1}{c} \sum_{i}\left\{f_{n} T_{0} \times f\left(S_{i}\right) \times \Delta S \times S_{i}^{b}\right\} \\
& =\frac{1}{c} f_{n} T_{0} \sum_{i}\left\{f\left(S_{i}\right) \times \Delta S \times S_{i}^{b}\right\} .
\end{aligned}
$$

Changing this into integral form can be expressed as follows:

$D_{\mathrm{f}}=\frac{1}{c} f_{n} T_{0} \int_{0}^{\infty} S^{b} f(S) \mathrm{d} S$.

By integrating this probability density function of the Rayleigh distribution, the cumulative fatigue damage $D_{\mathrm{f}}$ can be rewritten as follows by using the gamma function $\Gamma$ :

$D_{\mathrm{f}}=\frac{f_{n} T_{0}}{c}\left[\sqrt{2} \sigma_{S}\right]^{b} \Gamma\left(1+\frac{b}{2}\right)$.

Stress standard deviation $\sigma_{S}$ can be replaced by the velocity response root mean square (rms) value $\sigma_{v}$ according to the relationship in Eq. (3). According to this, Eq. (29) can be rewritten as follows:

$D_{\mathrm{f}}=\frac{f_{n} T_{0}}{c}\left[\sqrt{2} k \sigma_{v}\right]^{b} \Gamma\left(1+\frac{b}{2}\right)=f_{n} T_{0}\left(\frac{k^{b}}{c}\right)\left[2 \sigma_{v}^{2}\right]^{\frac{b}{2}} \Gamma\left(1+\frac{b}{2}\right)$.

By changing the symbols for $\sigma_{v}$ and rearranging, the formula to express the velocity response standard deviation $\sigma_{\mathrm{FDS}}\left(f_{n}\right)$ harmonizing to calculated $\operatorname{FDS}\left(f_{n}\right)$ by Eq. (18) can be obtained:

$\sigma_{\mathrm{FDS}}\left(f_{n}\right)=\sqrt{\frac{1}{2}\left[\frac{\operatorname{FDS}\left(f_{n}\right)}{f_{n} T_{0}\left(\frac{k^{b}}{c}\right) \Gamma\left(1+\frac{b}{2}\right)}\right]^{\frac{2}{b}}}$.

As mentioned above, the coefficients are set to $k=1$ and $c=1$, and by substituting the values of the parameters, $\sigma_{\mathrm{FDS}}\left(f_{n}\right)$ can be obtained.

According to well-known Miles' equation [21], power spectrum density (PSD) of stationary SPL can be obtained as follows:
Table 1 Summary of sample data

\begin{tabular}{ll}
\hline Parameters & Value \\
\hline Sampling rate, $F_{S}$ & $9600 \mathrm{~Hz}$ \\
Time span, $T$ & 0 (lift- \\
& off)-100 s \\
& (SRB burn \\
High-pass cut-off frequency, $f_{\mathrm{c}}$ & out) \\
\hline
\end{tabular}

$\operatorname{PSD}_{\mathrm{p}}\left(f_{n}\right)=\frac{8 \pi f_{n}}{Q\left(C_{\mathrm{np}} \frac{S_{a}}{m}\right)} \sigma_{v}\left(f_{n}\right)^{2}$.

By inserting Eqs. (24) and (31) into (32), stationary spectra $\operatorname{PSD}_{\mathrm{p}}\left(f_{n}\right)$ equivalent to non-stationary acoustic environment based on ERS and FDS can be obtained.

As the mentioned above, in general, it cannot be satisfied by one spectrum to be equivalence of the extreme stress and cumulative fatigue simultaneously because the extreme stress and cumulative fatigue are calculated in different formula. Therefore, there is an option to produce two test conditions for each different purpose of acoustic tests, extreme stress test and cumulative fatigue test, respectively, as stated in SMC standard [8]. Alternatively, conservative test conditions can be generated by selecting the maximum of $\sigma_{\mathrm{ERS}}$ and $\sigma_{\mathrm{FDS}}$ as follows:

$\sigma_{v}\left(f_{n}\right)=\max \left[\sigma_{\mathrm{ERS}}\left(f_{n}\right), \sigma_{\mathrm{FDS}}\left(f_{n}\right)\right]$.

\section{Application examples to flight telemetry}

In this section, application examples of the proposed method for measured flight telemetry are given.

\subsection{Liquid-propellant launch vehicle data}

The sound pressure telemetry of the liquid-propellant launch vehicle with SRB developed by JAXA shown in Fig. 2 was used for the analysis. The summary of this sample data is shown in Table 1. The upper limit frequency of analysis is $1 \mathrm{kHz}$, which is about $1 / 10$ of sampling rate to obtain accurate peak value according to the recommendation [22].

The comparison results of ERS and FDS between the flight acoustic environment, the conventional maximax spectra, and the proposed method are shown in Figs. 5 and 6 $\left(T_{0}=60 \mathrm{~s}\right.$ is adopted as a general acoustic test duration in the space industry (see e.g., $[1-3,23]$ ).

From Fig. 5, it can be seen that the maximax spectra at any time window length are different from the flight environment in both ERS and FDS. In contrast, according to Fig. 6, it can be confirmed that the proposed spectra are able 


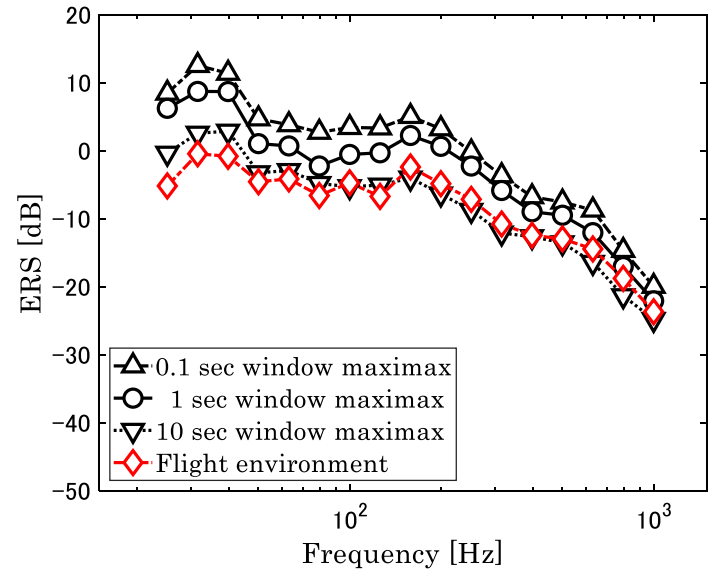

(a) ERS computation results $(0 \mathrm{~dB}=1 \mathrm{~m} / \mathrm{s})$

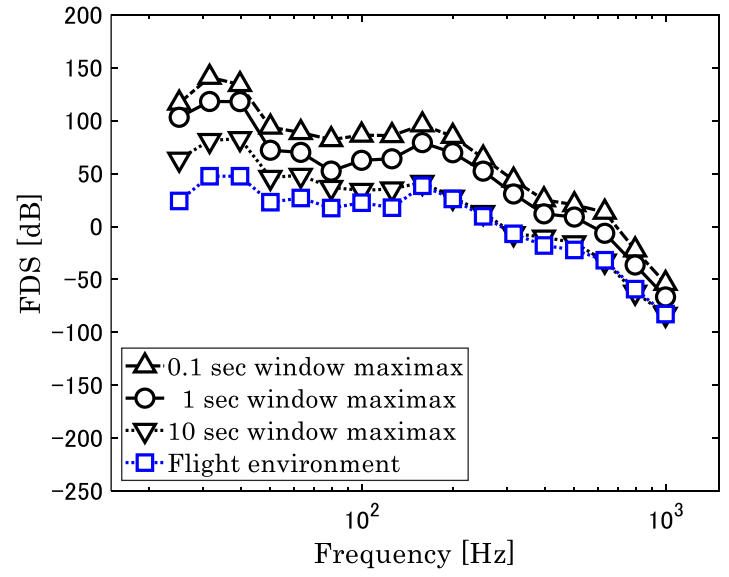

(b) FDS computation results $(b=6)(0 \mathrm{~dB}=1)$

Fig. 5 Comparison results of ERS and FDS between the flight acoustic environment and the maximax spectra

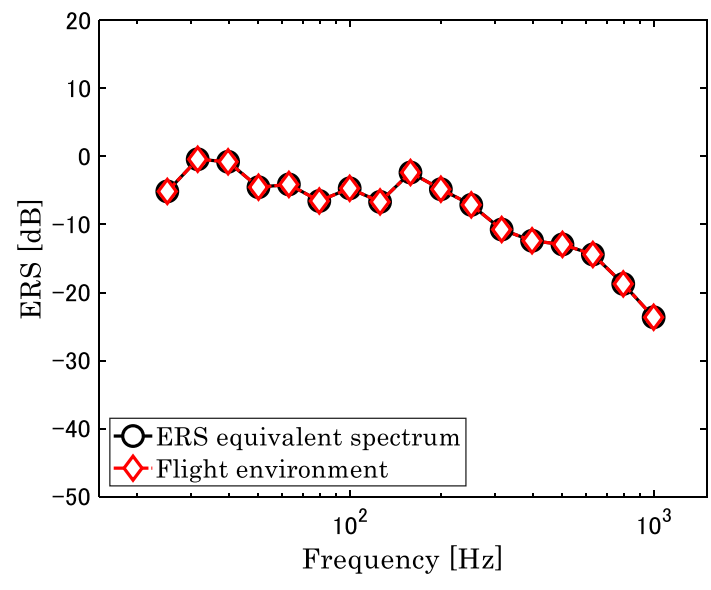

(a) ERS computation results $(0 \mathrm{~dB}=1 \mathrm{~m} / \mathrm{s})$

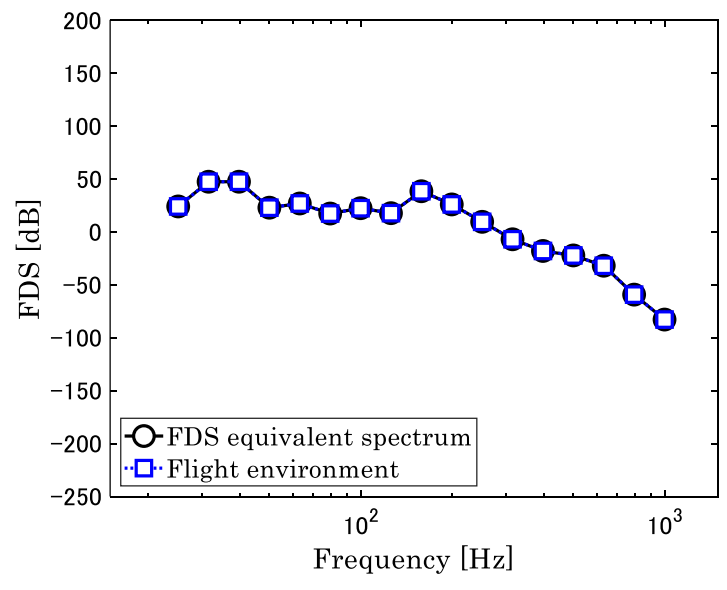

(b) FDS computation results $(b=6)(0 \mathrm{~dB}=1)$

Fig. 6 Comparison results of ERS and FDS between the flight acoustic environment and the proposed spectra

to make the ERS and FDS equivalent to the flight environment. Therefore, it can be said that a stationary acoustic test condition derived based on the proposed method can be equivalent to a flight non-stationary acoustic environment for structural vibro-acoustic response compared with the maximax spectrum.

Comparison results of stationary acoustic test condition between the maximax spectra and the proposed equivalent spectra are shown as Fig. 7.

From this application example of liquid-propellant launch vehicle, we find the following:
- The spectrum required for the extreme stress (ERS) is larger than that for the cumulative fatigue (FDS), and the O.A. value of the ERS equivalent spectrum $(\alpha=0.95)$ is $129.7[\mathrm{~dB}]$, which is about 3.3[dB] larger than 126.4 [dB] of the FDS equivalent spectrum $(b=6)$.

- ERS equivalent spectrum ( $\alpha=0.95$, O.A.: $129.7 \mathrm{~dB})$ is about $2.9 \mathrm{~dB}$ lower than the maximax spectrum of $1.0 \mathrm{~s}$ time window length (O.A.: $132.6 \mathrm{~dB}$ ), and FDS equivalent spectrum ( $b=6$, O.A.: $126.4 \mathrm{~dB}$ ) is about $6.2 \mathrm{~dB}$ lower. 
Fig. 7 Comparison results of stationary acoustic test spectra and the proposed spectra based on ERS $(\alpha=0.95)$ and FDS $(b=6)$ (liquid-propellant launch vehicle telemetry) condition between the maximax

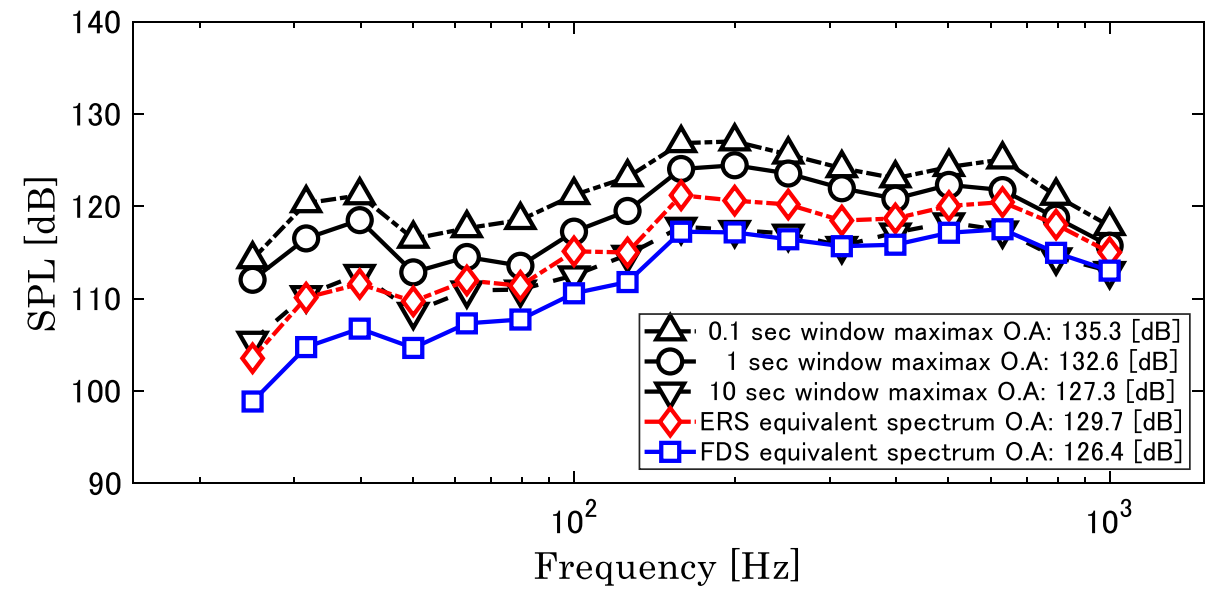

Fig. 8 Example of sound pressure telemetry of solid-propellant launch vehicle
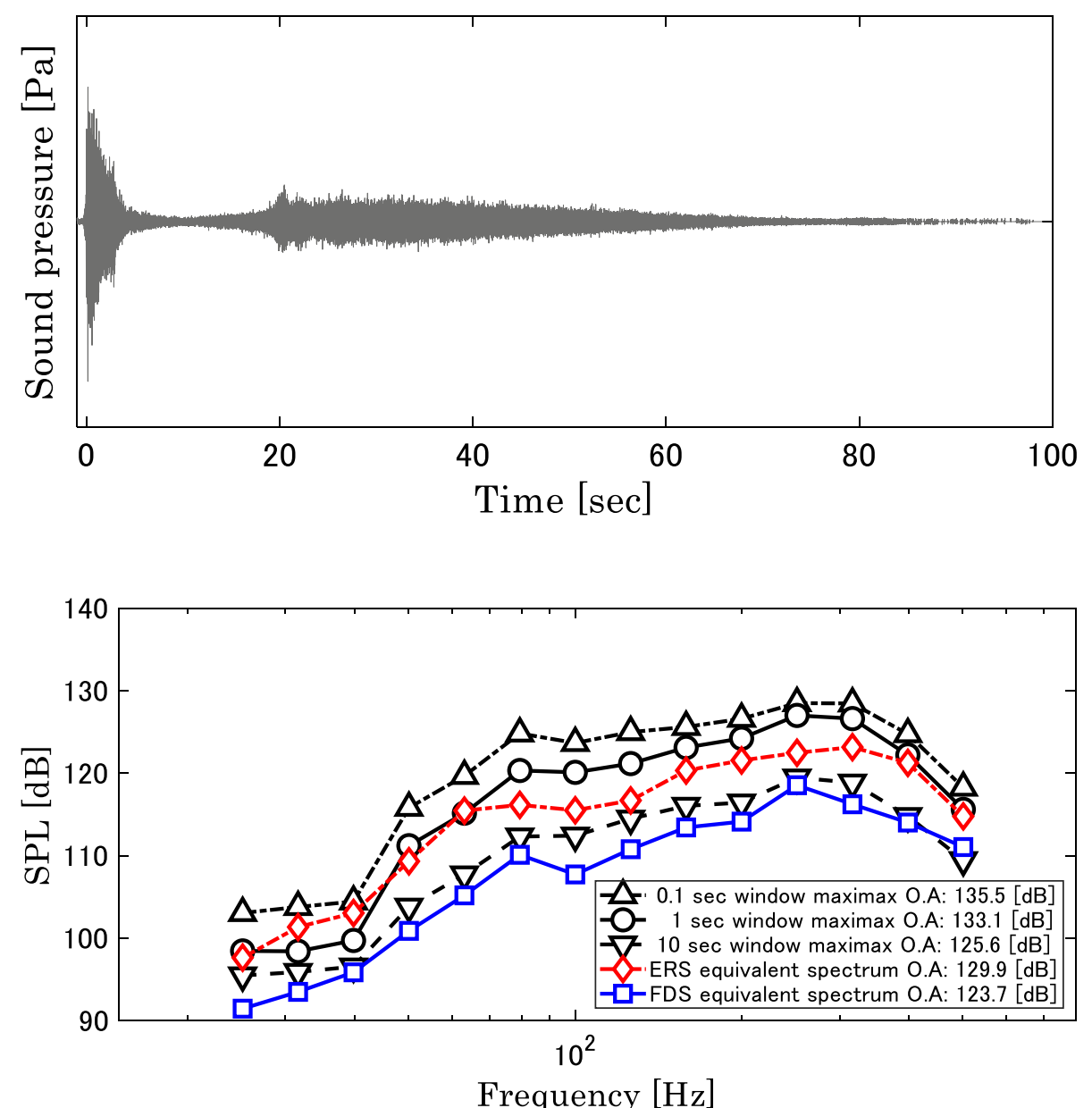

Fig. 9 Comparison results of stationary acoustic test condition between the maximax spectra and the proposed spectra based on ERS $(\alpha=0.95)$ and FDS $(b=6)$ (solid-propellant launch vehicle telemetry)

\subsection{Solid-propellant launch vehicle data}

Another application example, sound pressure telemetry of solid-propellant launch vehicle developed by JAXA is shown in Fig. 8. It can be seen by comparing with time history of liquid-propellant launch vehicle as shown in Fig. 2 that the sound pressure of transonic is lower than that after lift-off (Note that, the vertical axis range is different from that of Fig. 2). The computation results of this flight telemetry by the proposed method are shown in Fig. 9. The upper limit frequency of analysis is $500 \mathrm{~Hz}$ because the sampling rate of this time history is $4.8 \mathrm{kHz}$.

From this application example of solid-propellant launch vehicle, we find the following: 


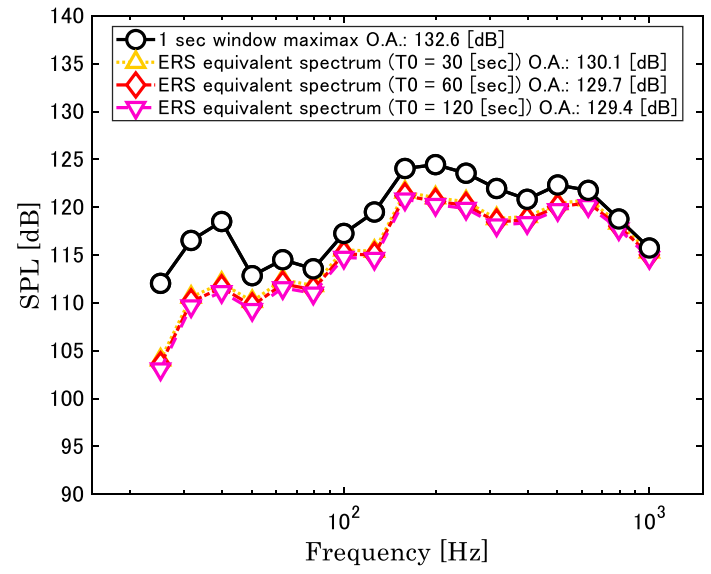

(a) Computation results for each $T_{0}(\alpha=0.95)$

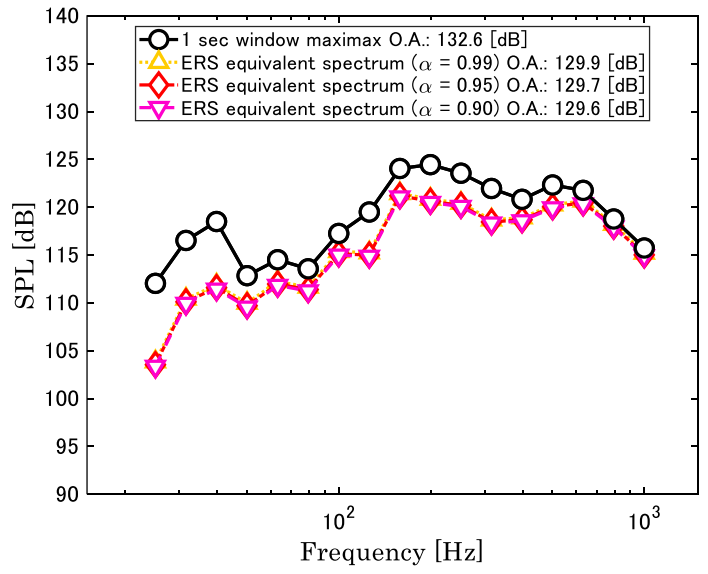

(b) Computation results for each $\alpha\left(T_{0}=60\right)$

Fig. 10 Computation results of ERS equivalent spectrum for different test duration $T_{0}$ (a) and probability threshold $\alpha(\mathbf{b})$

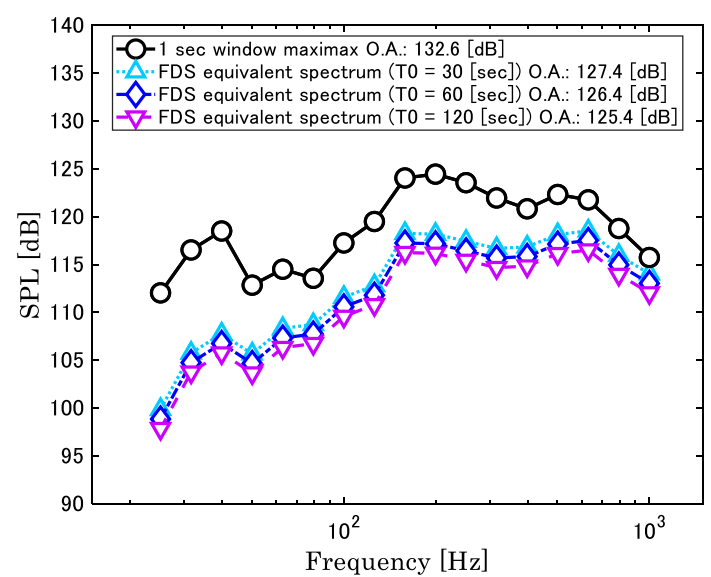

(a) Computation results for each $T_{0}(b=6)$

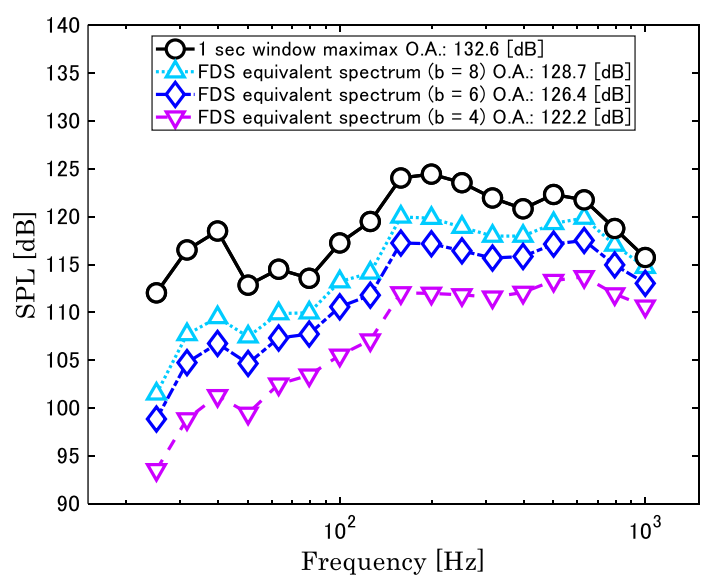

(b) Computation results for each $b\left(T_{0}=60\right)$

Fig. 11 Computation results of FDS equivalent spectrum for different test duration $T_{0}$ (a) and fatigue coefficient $b$ (b)

- The spectrum required for the extreme stress (ERS) is larger than that for the cumulative fatigue (FDS), and the O.A. value of the ERS equivalent spectrum $(\alpha=0.95)$ is $129.9[\mathrm{~dB}$ ], which is about $6.2[\mathrm{~dB}$ ] larger than $123.7[\mathrm{~dB}$ ] of the FDS equivalent spectrum $(b=6)$.

- ERS equivalent spectrum ( $\alpha=0.95$, O.A.: $129.9 \mathrm{~dB})$ is about $3.2 \mathrm{~dB}$ lower than the maximax spectrum of $1.0 \mathrm{~s}$ time window length (O.A.: $133.1 \mathrm{~dB}$ ), and FDS equivalent spectrum ( $b=6$, O.A.: $123.7 \mathrm{~dB})$ is about $9.4 \mathrm{~dB}$ lower. This relatively large difference can be understood from the fact that the sound pressure at transonic is smaller than that at lift-off, compared to the application example of liquid-propellant launch vehicle.

\section{Investigation of applied parameters}

The computation examples in previous section use $T_{0}=60, \alpha=0.95$ for ERS, and $b=6$ for FDS. In this section, the sensitivities to the stationary acoustic test conditions, computed by the proposed method when using different parameters, areas investigated. 
Table 2 Launch vehicles configuration used for analysis

\begin{tabular}{|c|c|c|c|c|c|}
\hline & & Trajectory type & & $\begin{array}{l}\text { SRB bul } \\
\text { launch) }\end{array}$ & out time (after \\
\hline Flight A & & $\mathrm{SSO} \rightarrow \mathrm{LEO}$ & & $91 \mathrm{~s}$ & \\
\hline Flight B & & LEO & & $99 \mathrm{~s}$ & \\
\hline Flight C & & EEO & & $99 \mathrm{~s}$ & \\
\hline Flight D & & SSO & & $115 \mathrm{~s}$ & \\
\hline Flight E & & LEO & & $99 \mathrm{~s}$ & \\
\hline Flight $\mathrm{F}$ & & $\mathrm{GTO} \rightarrow \mathrm{QZO}$ & & $97 \mathrm{~s}$ & \\
\hline Flight $\mathrm{G}$ & & EEO & & $115 \mathrm{~s}$ & \\
\hline Flight $\mathrm{H}$ & & SSO & & $116 \mathrm{~s}$ & \\
\hline \multicolumn{6}{|l|}{ ※1 } \\
\hline SSO: & Sun-Synchronous Orbit & & LEO: & & Low Earth Orbit \\
\hline EEO: & Earth Elliptical Orbit & & QZO: & & Quasi-Zenith Orbit \\
\hline GTO: & Geostationary Transfer Orbit & & & & \\
\hline
\end{tabular}

Fig. 12 The individual maximax spectra and their MPE spectrum $\left(T_{\mathrm{w}}=1.0\right.$, overlaps $=50 \%$ )

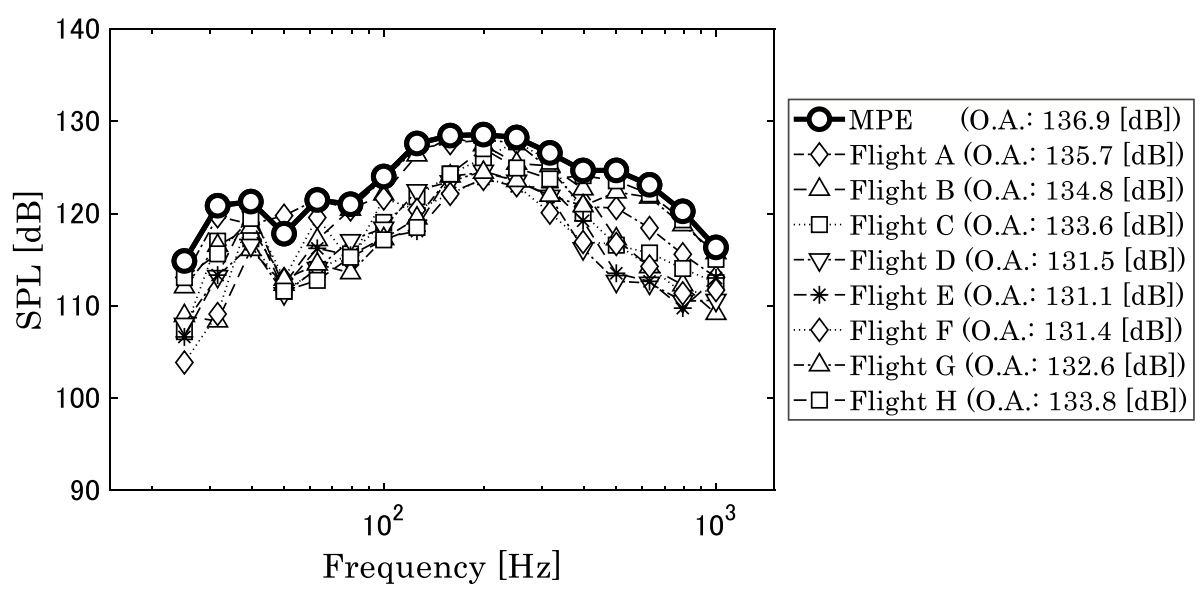

Figures 10 and 11 are the computation results of the stationary acoustic test conditions for the flight telemetry in Fig. 2 when using various parameters $\left(T_{0}, \alpha\right.$ and $\left.b\right)$.

Figures 10 and 11 can be summarized as follows:

- The ERS equivalent spectra and FDS equivalent spectra by changing $T_{0}$ when fixing $\alpha=0.95$ and $b=6$ are shown in Figs. 10a and 11a, it can be seen that the amount of change is smaller enough to be negligible for both ERS and FDS in case of $T_{0}=30-120 \mathrm{~s}$ (about 1-2 dB). This can be understood from Eqs. (24) and (31), which show less sensitivity to parameter $T_{0}$.

- The ERS equivalent spectrum per $\alpha$ when fixing $T_{0}=60$ $\mathrm{s}$ as shown in Fig. 10b, it can be seen that the amount of change is also small enough to be negligible in case of $\alpha=0.90-0.99$ (about $0.3 \mathrm{~dB}$ ), as indicated in Eq. (24) which shows less sensitivity to parameter $\alpha$.
- In contrast to $T_{0}$ and $\alpha$, FDS equivalent spectrum depends greatly on the value of $b$ and changes about $6.5 \mathrm{~dB}$ when $b$ changes from 4 to 8 , as shown in Fig. 11b. This can be understood from the fact that $b$ is a parameter that accelerates fatigue damage with stress magnitude, according to Miner's rule in Eq. (15).

As a conclusion from this parameters study, the ERS equivalent spectrum does not change significantly on parameters of probability $\alpha$ and test duration $T_{0}$, while the FDS equivalent spectrum changes significantly on parameter of fatigue coefficient $b$. For the selection of the fatigue coefficient $b$, it is desirable to apply the recommended design value in each space organization (e.g., $b=6$ is uniformly adopted for any types equipment of spacecraft by JAXA [23]). 
Fig. 13 The individual ERS equivalent spectra and their MPE spectrum $(\alpha=0.95)$

Fig. 14 The individual FDS equivalent spectra and their MPE spectrum $(b=6)$

Fig. 15 Comparison results of MPE based on the maximax spectrum and the proposed method
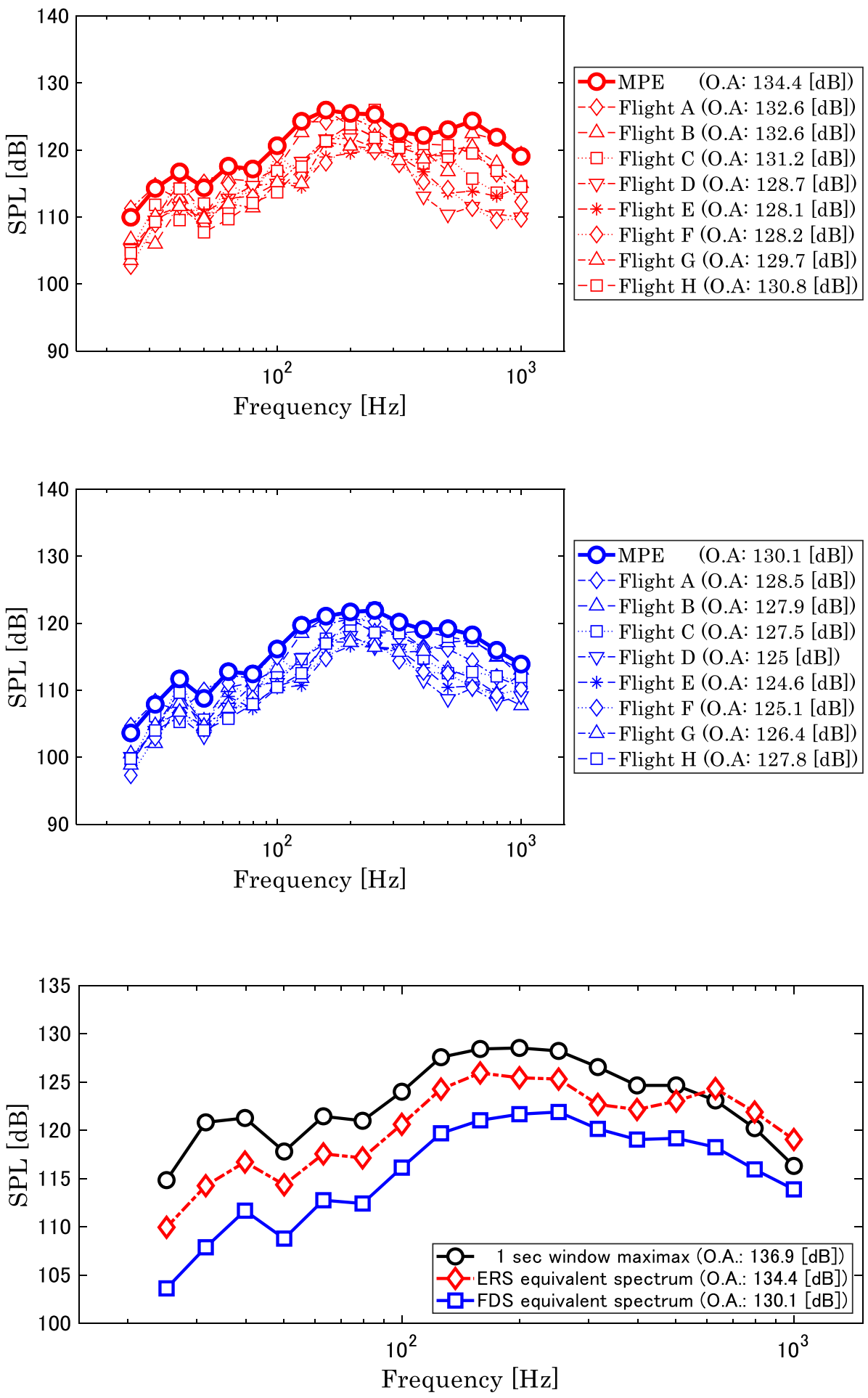

\section{Comparison of maximum predicted environment}

Ground acoustic test condition is generally a statistical maximum value with $\mathrm{P} 95 / 50^{1}$ as an envelope which counts

\footnotetext{
1 The P95/50 level is defined as enveloping 95 percent of the data with a 50 percent confidence level [24].
}

uncertainties of launch vehicles payloads and flight courses, etc., as the maximum predicted environment (MPE) (see, e.g., [23, 24]). The MPE is calculated by both the proposed method and the conventional maximax spectrum with flight telemetry of 8 liquid-propellant launch vehicles developed by JAXA. Launch vehicles configuration used for analysis are summarized in Table 2, and the analysis results are 
shown in Figs. 12, 13 and 14. Note that, for the computation of the maximax spectrum, $T_{\mathrm{w}}=1.0 \mathrm{~s}$ and overlaps $=50 \%$, which are commonly used in the space industry, were adopted (see e.g., [8, 23]).

From Fig. 12, the individual spectra by the maximax method varies from about 131-137 dB, and their MPE spectrum is $136.9 \mathrm{~dB}$. Moreover, from Figs. 13 and 14, the individual ERS equivalent spectra varies from about $128-133 \mathrm{~dB}$, and their MPE spectrum is about $134.4 \mathrm{~dB}$, and the individual FDS equivalent spectra varies from about $125-129 \mathrm{~dB}$, and their MPE spectrum is $130.1 \mathrm{~dB}$. The comparison results of the MPE spectra are shown in Fig. 15. MPE of ERS equivalent spectrum is about $2.5 \mathrm{~dB}$ and the FDS equivalent spectrum is about $6.8 \mathrm{~dB}$ lower than that of maximax spectrum with $T_{\mathrm{w}}=1.0 \mathrm{~s}$ and overlaps $=50 \%$.

As a conclusion, the statistically obtained stationary acoustic test condition (MPE) of liquid-propellant launch vehicle can be mitigated by this proposed method for both extreme stress and cumulative fatigue, especially more on fatigue damage spectrum, about $6.8 \mathrm{~dB}$ in this application example.

\section{Conclusions}

This paper proposed a new method to specify a stationary acoustic test condition based on ERS and FDS to replace the conventional maximax spectrum from the viewpoint of equivalence to structural vibro-acoustic response under a non-stationary and random flight acoustic environment. By specifying two independent acoustic specifications ERS and FDS, it may provide some option for acoustic and random vibration test. One option is two-phase acoustic or random vibration test, mentioned in reference [3] and [8]. For example, if the shock specification of a hardware is higher than ERS in critical mode frequency, strength verification of maximum load may be provided by shock test. In this case, acoustic or random vibration tests only may need to be verified for cumulative fatigue by using FDS.

As a result of applying the proposed method to the flight telemetry of the liquid- and solid-propellant propellant launch vehicles developed by JAXA, the following findings were obtained:

- The proposed method showed the effectiveness of mitigating acoustic test conditions for both liquid- and solid-propellant launch vehicles while maintaining the equivalence of the structural vibro-acoustic response, compared to the maximax spectrum with time window length $T_{\mathrm{w}}=1.0 \mathrm{~s}$ and overlaps $=50 \%$. Especially, the mitigation effect of the cumulative fatigue condition is significant for solid-propellant launch vehicles, where the sound pressure in the long-duration transonic phase is probably lower than that of short duration lift-off phase.

- The ERS equivalent spectrum does not change significantly on parameters of probability $\alpha$ and test duration $T_{0}$, while the FDS equivalent spectrum changes significantly on parameter of fatigue coefficient $b$. For the selection of the fatigue coefficient $b$, it is desirable to apply the recommended design value in each space organization.

- The statistically obtained stationary acoustic test condition (MPE) of liquid-propellant launch vehicles by the proposed method is shown to be effective in mitigating the extreme stress and cumulative fatigue by about 2.5 and $6.8 \mathrm{~dB}$, respectively. However, the value of mitigation of FDS may be dependent on applied fatigue acceleration coefficient.

Funding None.

Availability of data and material The datasets generated and/or analysed during the current study are not publicly open because most of them were JAXA's original flight data. The data are, however, available from the authors upon reasonable request and with permission of related JAXA contractors.

Code availability Not applicable.

\section{Declarations}

Conflict of interest None.

Open Access This article is licensed under a Creative Commons Attribution 4.0 International License, which permits use, sharing, adaptation, distribution and reproduction in any medium or format, as long as you give appropriate credit to the original author(s) and the source, provide a link to the Creative Commons licence, and indicate if changes were made. The images or other third party material in this article are included in the article's Creative Commons licence, unless indicated otherwise in a credit line to the material. If material is not included in the article's Creative Commons licence and your intended use is not permitted by statutory regulation or exceeds the permitted use, you will need to obtain permission directly from the copyright holder. To view a copy of this licence, visit http://creativecommons.org/licenses/by/4.0/.

\section{References}

1. National Aeronautics and Space Administration (NASA).: Dynamic environmental criteria, NASA-HDBK-7005, pp. 5-16, pp. 11-12 (2001)

2. European Space Aency (ESA).: Space engineering-testing, ECSS-E-10-03A, p. 15 (2002)

3. Military Standard.: Test requirements for launch, upper-stage, and space vehicles, MIL-STD-1540E, p. 111 (2006) 
4. ISO 19924.: Space systems—acoustic testing, https://www.iso. org/obp/ui/\#iso:std:iso:19924:ed-1:v1:en (2017)

5. Himelblau, H., Piersol, A.G., Wise J.H., Grundvig M.R.: Handbook for dynamic data acquisition and analysis. Institute of Environmental Science, IES Recommended Practice DTE012.1, Appendix B, pp. 287-302 (1996)

6. Rubin, S.: Damage-based analysis tool for flight vibroacoustic data. In: Proceedings of the 19th Aerospace Testing Seminar, pp. 190-203 (2000)

7. DiMaggio, S.J., Sako, B.H., Rubin, S.: Analysis of nonstationary vibroacoustic flight data using a damage-potential basis, J. Spacecr. Rockets 40(5) (2003)

8. Space and Missile systems Center (SMC).: Test requirements for launch, upper-stage and space vehicles SMC-S-016, p. 10, pp. 121-132 (2014)

9. Smallwood, D.O.: An improved recursice formula for calculating shock response spectra, shock and vibration bulletin. In: Proceedings of the 51st Symposium on Shock and Vibration (1980)

10. Lalanne, C.: Maximum response and fatigue damage spectraPart I. J. Environ. Sci. 35-40 (1984)

11. Lalanne, C.: Maximum response and fatigue damage spectraPart I. J. Environ. Sci. 40-44 (1984)

12. Lalanne, C.: Specification Development, vol. 5, 2nd edn. ISTE Ltd (2009)

13. Henderson, G.R., Piersol, A.G.: Fatigue damage related descriptor for random vibration test environments, sound and vibration, $p p$. 20-24 (1995)

14. McNeill, S.I.: Implementing the fatigue damage spectrum and fatigue damage equivalent vibration testing. In: The 79th Shock and Vibration Symposium, pp. 1-20 (2008)
15. Cho, D.H.: Evaluation of vibration test severity by FDS and ERS, pp. 1725-1736. In: Proceedings of ISMA (2010)

16. Hunt, F.V.: Stress and strain limits on the attainable velocity in mechanical vibration. J. Acoust. Soc. Am. 32, 1123 (1960)

17. Ando, S., Yanagase, K., Shi, Q.: Simplified vibroacoustic analysis of structure subjected to diffuse sound field excitation using joint acceptance. Trans. Jpn. Soc. Mech. Eng. Ser. C 76(764), 818-824 (2010). (in Japanese)

18. MathWorks: Signal Processing Toolbox. https://jp.mathworks. $\mathrm{com} / \mathrm{help} /$ signal/ref/rainflow.html?lang=en

19. Miner, M.A.: Cumulative damage in fatigue. J. Appl. Mech. 12, 149-164 (1945)

20. Harris, C.M., Piersol, A.G.: HARRIS' Shock and Vibration Handbook, CHAPTER 11, 5th edn., p. 11.1-11.3. McGraw-Hill (2002)

21. Miles, J.W.: On structural fatigue under random loading. J. Aeronaut. Sci. 753 (1954)

22. Ahlin, K.: Shock response spectrum calculation-an improvement of the Smallwood Algorithm. In: 70th Shock and Vibration Symposium, November (1999)

23. Japan Aerospace Exploration Agency (JAXA).: Acoustic test handbook, JERG-2-130-HB003B, pp. A-3-A-6 (2017) (in Japanese)

24. National Aeronautics and Space Administration (NASA).: Payload vibroacoustic test criteria, NASA-STD-7001, p. 27, pp. 31-32 (2011)

Publisher's Note Springer Nature remains neutral with regard to jurisdictional claims in published maps and institutional affiliations. 